\title{
Underwater tunable organ-pipe sound source
}

\author{
Andrey K. Morozov a) and Douglas C. Webb \\ Webb Research Corporation, 82 Technology Park Drive, East Falmouth, Massachusetts 02536
}

(Received 24 July 2006; revised 18 January 2007; accepted 31 May 2007)

\begin{abstract}
A highly efficient frequency-controlled sound source based on a tunable high- $Q$ underwater acoustic resonator is described. The required spectrum width was achieved by transmitting a linear frequency-modulated signal and simultaneously tuning the resonance frequency, keeping the sound source in resonance at the instantaneous frequency of the signal transmitted. Such sound sources have applications in ocean-acoustic tomography and deep-penetration seismic tomography. Mathematical analysis and numerical simulation show the Helmholtz resonator's ability for instant resonant frequency switching and quick adjustment of its resonant frequency to the instantaneous frequency signal. The concept of a quick frequency adjustment filter is considered. The discussion includes the simplest lumped resonant source as well as the complicated distributed system of a tunable organ pipe. A numerical model of the tunable organ pipe is shown to have a form similar to a transmission line segment. This provides a general form for the principal results, which can be applied to tunable resonators of a different physical nature. The numerical simulation shows that the "state-switched" concept also works in the high- $Q$ tunable organ pipe, and the speed of frequency sweeping in a high- $Q$ tunable organ pipe is analyzed. The simulation results were applied to a projector design for ocean-acoustic tomography. (C) 2007 Acoustical Society of America.
\end{abstract}

[DOI: $10.1121 / 1.2751268]$

PACS number(s): 43.30.Yj, 43.38.Ew, 43.30.Jx [JAC]

Pages: $777-785$

\section{INTRODUCTION}

Low-frequency broadband underwater sound sources are used in sonar systems, ocean-acoustic tomography, and seismic deep-penetration profiling systems. ${ }^{1-3}$ In these systems, various physical mechanisms are applied to low-frequency signal generation, such as pneumatic, electromagnetic, magnetostrictive, electrostatic, hydroacoustic, and parametric. A variety of underwater sound sources (including Tonpilz, Helmholtz resonator, flexural, and bubble transducer.) are described in the scientific literature. ${ }^{4-9}$ Sound-source design requires accounting for certain fundamental physical principles and problems that are inherent in underwater transducers that use arbitrary mechanisms of energy transformation. Emitted acoustic energy is proportional to a square volume velocity and frequency; to produce high-amplitude and lowfrequency signals, an underwater source must generate large volume displacement. As a result, a sound source with a small radiation area has a large imaginary part of impedance, bigger than its real part.

There are two potential approaches to building a projector with high efficiency and large radiated acoustic power. The first approach is to increase the amplitude of the displacement. This is accomplished in transducers by using flexible membranes, bars, or plates of large area and dimension. Such transducers require complicated and expensive techniques and in most cases require pressure compensation.

Another option is to use high- $Q$ resonators near the resonance frequency. The reactive part of the resonator in resonance with the radiated signal eliminates the reactance of the radiation impedance. A high ratio of radiation reactance to

${ }^{a)}$ Electronic mail: moro@webbresearch.com the real part of the radiation impedance suggests use of a high $Q$ of the resonator. Practical experience and common sense tell us that high- $Q$ resonant sources should have high efficiency, relatively smaller dimensions, and uncomplicated design. A narrow-frequency bandwidth is the only disadvantage of such resonant sound sources. Application of the tunable or switchable high- $Q$ resonant sound source is one way to generate signals, which occupy a very large frequency bandwidth. The projector need not produce a linear, timeinvariant broadband transformation of input signals to generate a broadband acoustic wave field. The sound source does not have to emit all components of a broadband signal simultaneously, as is necessary for linear time invariant systems. A high- $Q$ narrow-band resonant system can generate a broadband signal if it is tuned synchronically with the instantaneous signal frequency and always maintained in a resonance state. Moreover, it is possible to use both a quick (instant) switch of a resonance frequency simultaneously with an instantaneous signal frequency shift and slow tracking of the signal frequency. As noted by Larson et al., ${ }^{10}$ Munk proposed this method in 1980 as a "state-switched" sound source concept when he was analyzing different approaches for design of high-efficiency sound sources for an ocean-acoustic tomography experiment. The "state-switched acoustic source" can switch among several different resonant states instantly and synchronically with the discrete frequency manipulated signal. If the source at any moment in time has only one fundamental resonant frequency and always maintains resonance with the signal, it radiates a highly efficient acoustic wave. Reference 10 describes this concept in detail, with the example of a simple mass-spring harmonic oscillator and a description of a prototype underwater stateswitched sound source with two states, 810 and $1022 \mathrm{~Hz}$. 
Although a variety of designs for high-frequency stateswitched sources is described in the literature, ${ }^{11-18}$ there is no good design for a state-switched transducer with a frequency smaller than $800 \mathrm{~Hz}$. The state-switched transducer adequate for frequency coded signal transmission is used in communications and underwater sonar. Many applications require neither complex, coded-signal transmission nor simultaneous transmission of all broadband acoustic-energy spectrum components. Different frequencies transmitted in sequence, or swept signals, are appropriate when the medium under investigation does not change appreciably over the duration of the transmission. In that case, one can use relatively slow resonance frequency tuning of a high- $Q$ system corresponding to the instantaneous frequency of the linear frequencymodulated signal. The swept frequency modulation does not degrade the time resolution. The Cramer-Rao bound for time resolution for a system operating above threshold and using a matched filter is $\sigma_{\Delta T}=1 /(\sqrt{\mathrm{SNR} W})$, where SNR is the ratio of total received energy to a spectral level of noise, and $W$ is the total system bandwidth, not an instantaneous one, and the only implications of a FM signal is the range-Doppler coupling. In our analysis, we will distinguish total system bandwidth from the instantaneous bandwidth of narrow-band tunable resonator. The swept frequency transducer meets all the requirements of stationary systems for ocean monitoring, including ocean-acoustic tomography and seafloor monitoring. Three papers (Refs. 19-21) describe a frequency swept sound source based on a tunable organ pipe. It is a reliable, broadband, depth-independent, highly efficient sound source capable of long-term operation. This makes it superior to the marine vibroseis, ${ }^{22}$ which is less efficient, needs pressure gas compensation, and cannot be used at large depths.

A simple and practical model of a tunable organ pipe ${ }^{20}$ will be used further for simulation of the frequency and time responses of a tunable organ-pipe source.

This paper's objective is to consider general aspects of tunable resonant sound-source theory and to provide detailed analysis of time-response processes in the practical design of a real tunable organ pipe. The organization of the paper is as follows.

Section II provides theoretical analysis of the problem, beginning with consideration of a general concept for a "quick frequency adjustment" (QFA) filter. Suppose that a broadband signal with an arbitrarily changing phase narrow or wideband phase modulation is required on the output of a high- $Q$ filter with the bandwidth, which can be much smaller than signal spectrum bandwidth. A QFA filter is a resonant filter, whose resonant frequency coincides at any moment with the instantaneous frequency of the input signal; an example is a high- $Q$ tunable projector that maintains resonance with the transmitted signal at any given moment. Such a projector has high efficiency and all other advanced characteristics of high- $Q$ resonant systems along with a broadband radiated signal spectrum. The theory of QFA filters as considered here is based on the tunable Helmholtz resonator. Our research shows that a simple tunable Helmholtz resonator can work as a QFA filter: We show analytically and by numerical simulation that the simplest lumped element tun- able resonator can be successfully used as a state-switched sound source or as a frequency swept source.

In Secs. III and IV the analysis moves from the simplest lumped sound source to a complex distributed system, such as a tunable organ pipe. A numerical model of a real tunable organ pipe is developed. The electrical circuit model of the organ pipe has a form of a transmission line segment and is equivalent to high-frequency electromagnetic resonator models. This gives a general form to the results of the analysis. Computer simulation of an organ pipe conducted in Sec. IV shows that it can also be used as a highly efficient, stateswitched transducer or a frequency swept projector. The analysis is then applied to design of a real sweeping transducer for acoustic tomography and global ocean monitoring. Section V presents result of tunable organ pipe experimental testing. The projector described has been used in NPAL 2004 experiments in the Pacific Ocean.

Section VI offers the summary and conclusions.

\section{SIMPLEST SECOND-ORDER TUNABLE RESONANT CIRCUIT}

The ability to change resonance frequency quickly is a property of any lumped element resonant system of the second-order. Indeed, the solution for simplest resonant system described by the second-order differential equation is completely determined for any time from half-space $t \geqslant t_{0}$ by $x\left(t_{0}\right)$ and its derivative $x^{\prime}\left(t_{0}\right)=(d x / d t)\left(t_{0}\right)$. The system has no memory for the parameters or coefficients, and thus no memory for the resonance frequency. If the resonance frequency switches from $\omega_{1}$ to $\omega_{2}$, the oscillation instantly changes its wave form from $x(t)=A_{1} \sin \left(\omega_{1} t+\varphi_{1}\right)$ to $x(t)$ $=A_{2} \sin \left(\omega_{2} t+\varphi_{2}\right)$, where $A_{2}=\sqrt{x^{2}\left(t_{0}\right)+x^{\prime 2}\left(t_{0}\right) / \omega_{2}^{2}}$ and $\varphi_{2}$ $=\arctan \left(x\left(t_{0}\right) \omega_{2} / x^{\prime}\left(t_{0}\right)\right)-\omega_{2} t_{0}$ are amplitude and phase of a final oscillation. Note that if the state switches when $x\left(t_{0}\right)$ $=0$ or $x^{\prime}\left(t_{0}\right)=0$, then the phase does not change value, and amplitude jumps to the value $A_{2}=A_{1} \omega_{1} / \omega_{2}$; in all other cases, phase changes as well. To avoid additional phase manipulation during the frequency change, the original stateswitched concept expected instant resonance frequency switch only when $x\left(t_{0}\right)=0$ or $x^{\prime}\left(t_{0}\right)=0$. The amplitude signal hop still accompanies any frequency switching in accordance with $A_{2}=\sqrt{x^{2}\left(t_{0}\right)+x^{\prime 2}\left(t_{0}\right) / \omega_{2}^{2}}$. The frequency sweeping or chirp signal can be performed by the resonance frequency switching among several frequencies. ${ }^{10,11}$ All abovedescribed formulas were obtained from simple requirements for continuity of signal, and its derivative. It should be noted that different variants of that principle can have specific properties, but, in any case, the state-switched process conserves energy, and any energy changing in a high- $Q$ resonance system requires a slow transient process.

To analyze the general concept of a quick frequency adjustment filter, the system from Fig. 1 is considered. Suppose that a broadband signal with an arbitrarily changing phase is required on the output of a high- $Q$ tunable filter. The resonant frequency of the filter is controlled by computer to match the instantaneous frequency of the input signal at all times. The QFA filtering can be implemented using a phaselocked loop (PLL) to follow the changing frequency of the 


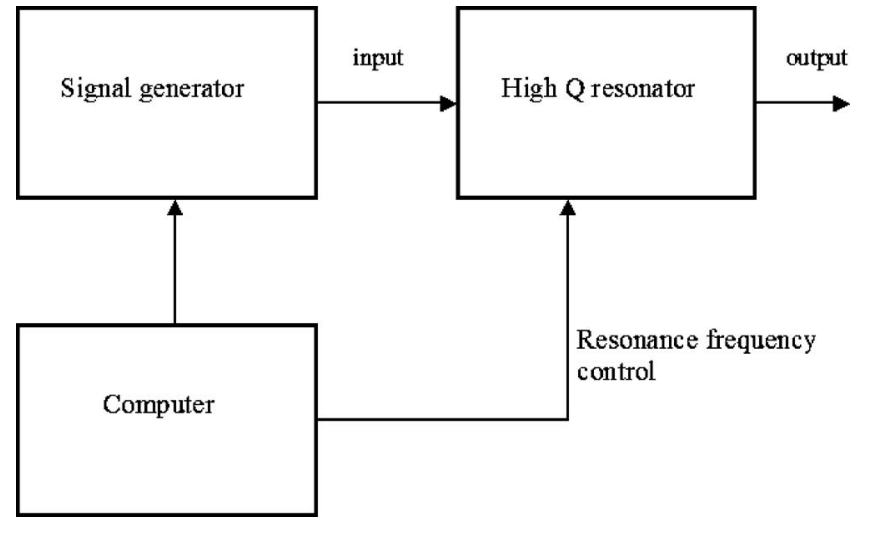

FIG. 1. The control system for QFA filter.

signal. The details of such a PLL are provided in Refs. 19-21 and are not included in this analysis. The objective of this paper is to demonstrate the ability of the QFA filter to reproduce a general broadband signal with an arbitrarily changing phase without constraints on the first- or second-order derivatives, that is, the rates of change, of that phase.

The differential equation for a simplest resonant circuit in a canonical form is

$$
\frac{d^{2} V}{d t^{2}}+2 \delta \omega_{r} \frac{d V}{d t}+\omega_{r}^{2} V=\omega_{r}^{2} V_{0},
$$

where $\delta=1 / 2 Q$ is the loss factor; $Q$ is the quality, or resonance, factor; and $\omega_{r}$ is the resonance frequency.

The Helmholtz resonator is the simplest example of a lumped element resonant system. Several papers describe tuning methods, ${ }^{11-18}$ and some examples of tunable Helmholtz resonators can be found in others (see Refs. 23-26). In the case of the Helmholtz resonator, the wave forms $V_{0}$ and $V$ are the volume velocities and other parameters of Eq. (1):

$$
\begin{aligned}
& \omega_{r}=c \sqrt{\frac{S}{l \Omega}}, \\
& Q=\frac{2 \lambda l}{S} .
\end{aligned}
$$

The Helmholtz resonator parameters are: $\Omega$ is the resonator volume, $S$ is the throat area, $l$ is the throat length, $\beta=1 / K$ is the compressibility of water, and $K$ is the bulk modulus.

Our analysis does not include detailed consideration of the tunable mechanism of the Helmholtz resonator. For example, the resonator can be tuned by opening a path to an additional container with a compressible liquid. As a result, the resonance frequency $\omega_{r}=\omega_{r}(t)$ will be variable. The objective is to show the potential ability of the Helmholtz resonator to quickly adjust to the signal with a variable instantaneous frequency. This ability allows it to radiate a broadband signal $V(t)=A \sin (\varphi(t))$ with the continuous instantaneous frequency $\omega(t)=d \varphi(t) / d t$ by a quick adjustment of the resonance frequency of the high- $Q$, narrow-band Helmholtz resonator.

The quick adjustment condition means that any time resonance frequency of the tunable resonator $\omega_{r}(t)$ is equal to the instantaneous signal frequency $\omega(t)$,

$$
\omega_{r}(t)=\omega(t)=\frac{d \varphi(t)}{d t} .
$$

The radiated signal derivative forms are

$$
\begin{aligned}
& \frac{d V(t)}{d t}=\frac{d \varphi(t)}{d t} A \cos (\varphi(t)), \\
& \frac{d^{2} V(t)}{d t^{2}}=\frac{d^{2} \varphi(t)}{d t^{2}} A \cos (\varphi(t))-\left(\frac{d \varphi(t)}{d t}\right)^{2} A \sin (\varphi(t)) .
\end{aligned}
$$

Substituting these formulas into the initial equation (1) and taking into account the adjustment frequency condition (4), the form of a drive signal $V_{0}(t)$ becomes

$$
\begin{aligned}
V_{0}(t) & =\left(\frac{d \varphi(t)}{d t}\right)^{-2}\left(2 \delta \omega_{r} \frac{d \varphi(t)}{d t}+\frac{d^{2} \varphi(t)}{d t^{2}}\right) A \cos (\varphi(t)) \\
& =A\left(2 \delta+\frac{1}{\omega^{2}(t)} \frac{d \omega(t)}{d t}\right) \cos (\varphi(t))
\end{aligned}
$$

We just proved that Eq. (1) with the right part in the form

$$
\omega_{r}^{2}(t) V_{0}(t)=A\left(2 \delta \omega_{r}^{2}(t)+\frac{d \omega(t)}{d t}\right) \cos (\varphi(t))
$$

has the solution $V(t)=A \sin (\varphi(t))$. The output of the QFA filter has the form $V(t)=A \sin (\varphi(t))$ with the arbitrarily changing phase $\varphi(t)$, if the input signal has the form of Eq. (6),

$$
V_{0}(t)=A\left(2 \delta+\frac{1}{\omega_{r}^{2}(t)} \frac{d \omega(t)}{d t}\right) \cos (\varphi(t)) .
$$

There is no limit on the derivatives of the first or second order, and no limitation on the phase spectrum bandwidth.

In the simplest $\mathrm{CW}$ case, when $V(t)=A \sin \left(\omega_{r} t\right)$, the wave form for $V_{0}(t)$ is

$$
V_{0}(t)=2 \delta A \cos \left(\omega_{r} t\right) \text {. }
$$

In the case of a linear frequency modulated signal (LFM), when $V(t)=A \sin \left(\omega_{0} t+0.5 a t^{2}\right)$, the wave form for $V_{0}(t)$ is

$$
V_{0}(t)=\left(2 \delta+a /\left(\omega_{0}+a t\right)^{2}\right) A \cos (\varphi(t)) .
$$

The output of the QFA filter in a form $V(t)=A \sin \left(\omega_{0} t\right.$ $\left.+0.5 a t^{2}\right)$ can be achieved by exciting it with the wave form in Eq. (8) with no limitation of the rate $a$. As result, the system bandwidth can be much larger than the instantaneous bandwidth of a high- $Q$ tunable system.

The amplitude of signal $V_{0}(t)$ in Eq. (6) has two components. The first component, $A \delta \omega^{-1}(t)$, is quasistationary and has the same form as in CW case. The second,

$$
A \frac{1}{\omega^{-2}(t)} \frac{d \omega(t)}{d t}
$$

is a changing of amplitude due to frequency changes during a time comparable with the period. That component can be positive (sweeping from low frequency to high frequency) or negative (sweeping from high frequency to low frequency). This component is essential only for very fast frequency 


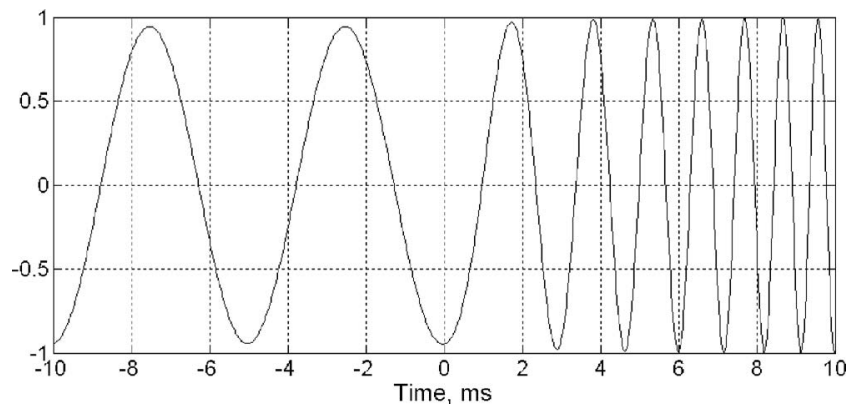

FIG. 2. The response of tunable Helmholtz resonator to fast frequency sweep from 200 to $1200 \mathrm{~Hz}$ for $10 \mathrm{~ms}$.

changes, when from period to period it is changed $20 \%$ or more.

The numerical simulation demonstrates the ability of the simplest lumped element resonator to quickly adjust its resonance frequency to an instantaneous frequency of a broadband signal. The response of a tunable Helmholtz resonator to a fast frequency sweep of $200-1200 \mathrm{~Hz}$ for $10 \mathrm{~ms}$ is shown in Fig. 2. Figure 3 presents the response to a frequency hop of $200-1200 \mathrm{~Hz}$ for the same resonator. The QFA concept allows the generation of very short broadband chirp signals and generalizes the above-mentioned switchedstate concept. The concept is an inherent property of secondorder lumped-element resonant circuits, but it also applies to more complicated circuits, such as the organ pipe discussed in the following as an example of a distributed acoustic system.

\section{TUNABLE RESONATOR TUBE}

References 18-20 describe a design for a tunable, resonant organ-pipe sound source. It is a very reliable projector with the ability to radiate swept-frequency signals with high efficiency, high power, and unlimited operating depth. The projector is a freely flooded, mechanically tunable organ pipe with a Tonpilz acoustical driver. A computer-controlled electrical actuator keeps the projector in resonance with the swept-frequency signal by means of phase-lock-loop feedback. This projector combines the efficiency and simplicity of resonant tube projectors with the possibility of using wide frequency ranges.

The organ-pipe design is a configuration of two slotted resonator tubes driven by a coaxially mounted, symmetrical Tonpilz transducer. To change the resonant frequency of the

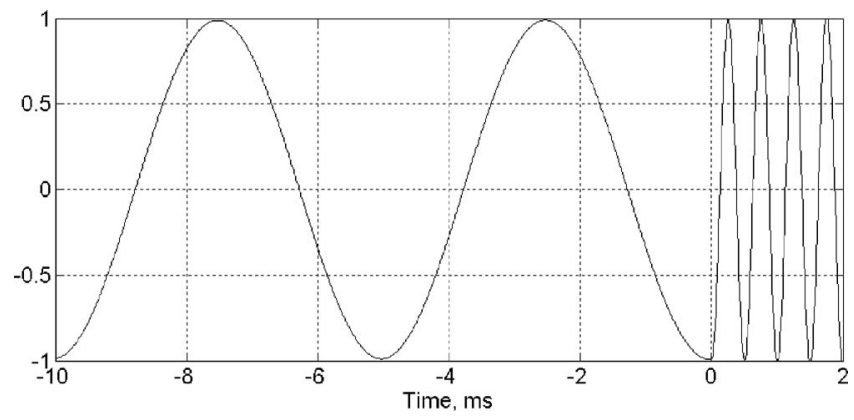

FIG. 3. The response of tunable Helmholtz resonator to instant frequency hop from 200 to $2000 \mathrm{~Hz}$.

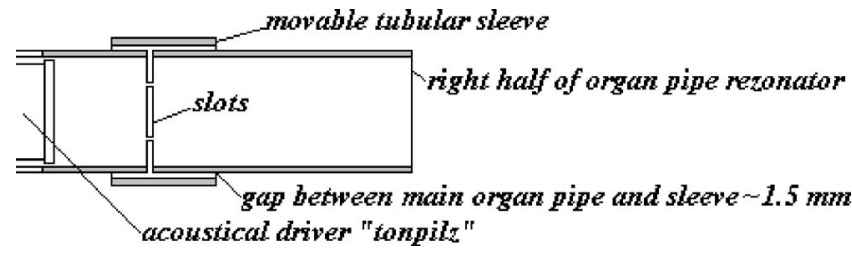

FIG. 4. Draft drawing of the tuning mechanism of organ pipe sound projector, the linear actuator is not shown.

projector, the resonator tubes were fitted with slots (or vents) at a distance of one-third the resonator tube length measured from the acoustical driver. ${ }^{27}$ Two stiff coaxial tubular sleeves of larger diameter move axially along the resonator tubes, changing the exposure of the slots (Fig. 4). The inertia of the water layer in the gap between the two coaxial tubes depends on the position of the sleeves relative to the tube slots. The position of the sleeves causes a change in the equivalent acoustic impedance of the slots, thus changing the resonant frequency. As a result, the resonant frequency varies with the position of the sleeves relative to the slots. A computercontrolled actuator moves the sleeves and keeps the projector in resonance with a swept-frequency signal.

A simplified equivalent electrical-circuit model can be successfully applied similar to the tunable organ-pipe resonant sound source. The model is based on the similarity of mechanical differential equations and equations for ordinary electrical elements, such as capacitors, inductors, resistors, and transformers. ${ }^{20}$ The model is simpler than the finite element analysis, ${ }^{20}$ and it does not need special software or powerful computers to facilitate the prediction of precise projector parameters. This model was continuously compared with experimental data from the actual projector test. The comparison showed that it truly reflects organ-pipe sound physics. In this model, we did not take into account the inertia of the aluminum pipe walls, the losses in the walls, the deformation of the Tonpilz transducer shell, the radiation from the orifice, or other small details of the actual projector performance.

Let us assume that a Tonpilz acoustical driver includes $m$ ceramic stacks in cylindrical form composed of $n$ piezoelectric ceramic longitudinally polarized cylinders. The entire area of the ceramic stacks is $A_{c}$. The length of one ceramic cylinder is $t_{c}$ and the length of all the stacks is $l_{c}$. The piezoelectric ceramic polarization direction (three by convention) is in the axial direction. The reduced constitutive relations ${ }^{19}$ for a piezoelectric ceramic are shown in the simple equations

$$
\begin{aligned}
& S_{3}=s_{33}^{E} T_{3}+d_{33} E_{3}, \\
& D_{3}=\varepsilon_{3}^{T} E_{3}+d_{33} T_{3},
\end{aligned}
$$

where $S_{3}$ is the three-strain component, $T_{3}$ is the three-stress component, $E_{3}$ is the electric field in the three directions, and $D_{3}$ is the electric displacement in the three directions. The piezoelectric material properties are given by compliance $s_{33}^{E}$, piezoelectric strain coefficient $d_{33}$, electric permittivity $\varepsilon_{3}^{T}$, and density $\rho_{c}$. The ceramic stack emits sound pressure with 


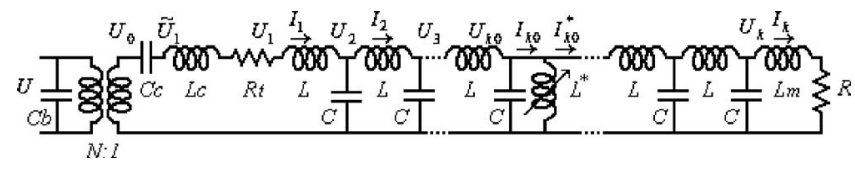

FIG. 5. Resonant organ pipe equivalent circuit.

a frequency $f$ into the water through a piston with area $A_{p}$, length $l_{p}$, and material density $\rho_{p}$.

The acoustic Tonpilz driver is placed between a pair of open-ended, cylindrical tubes with individual length $L$, crosssectional area $A$, and thickness $h$. Each section has orifices with the area $A_{o}$ formed in the walls at a distance $L_{o}$ from the acoustic driver. The movable coaxial sleeve has length $l_{s}$ and radius $g$, which is larger than the radius of the main tubular section. The projector tubes are freely flooded with water (density $\rho_{o}$ and sound velocity $c_{o}$ ).

Figure 5 shows the simplified electrical equivalent circuit for one-half of the symmetric resonator tubes, where $C_{b}=n A_{c} \varepsilon_{33}^{T}\left(1-k_{33}^{2}\right) / t_{c}$ is the capacity of the piezoelectric ceramic for a clamped circuit, $C_{b}=n A_{c} \varepsilon_{33}^{T} / t_{c}$ is the same capacity for an open circuit, $k_{33}=d_{33} / \sqrt{\varepsilon_{33}^{E} s_{33}^{E}}$ is the coupling coefficient (a property of the piezoelectric ceramic material), $C_{c}=l_{c} s_{33}^{E} A_{p}^{2} / A_{c}$ is the ceramics stiffness equivalent capacitor,

$$
L_{c}=\frac{4 \rho_{c} A_{c} l_{c}}{A_{p}^{2} \pi^{2}}+\frac{\rho_{p} l_{p}}{A_{p}}
$$

is the lumped equivalent inductance of the combined ceramics and piston inertia, $R_{t}=\left(1-A_{c} / A_{p}\right)^{2} \pi \rho_{o} f^{2} / c_{o}$ is the radiation resistance from the Tonpilz center, $N=d_{33} A_{c} /\left(A_{p} s_{33}^{E} t_{c}\right)$ is the transformation coefficient, $A_{p}$ is the piston area, $L$ $=\rho_{o} d / A$ is the inertia of the water mass in the tubular section with length $d, C=C_{\text {water }}+C_{\text {wall }}=A \beta d+2 A R_{a} d /(E h)$ is the combined capacitance of the wall stiffness and water compressibility, $\beta=1 /\left(c_{0} \rho_{0}\right)$ is the bulk modulus of water, $d$ $=L / k$ is the length of one section (where $k$ is the number of sections in the numerical model and $L$ is the length of the tube), $L^{*}$ is the variable inductance equivalent for the water inertia in the gap between the resonator tube and the movable sleeve, $L_{m}=0.25 \rho_{o} \sqrt{\pi / A}$ is the inductance of the added mass of the open resonator tube end, and $R=\pi \rho_{o} f^{2} / c_{o}$ is the radiation resistance. ${ }^{20}$

The key element of the projector design is the variable inductance $L^{*}$. The inductance is derived from the position of the movable sleeve relative to the position of the slot in the resonator tube. When the slot is completely uncovered, this inductance can be calculated from Eq. (10) for the added mass $m_{a}$ of the open orifice, which is

$$
m_{a}=0.5 \rho \sqrt{\pi} A_{o}^{3 / 2} .
$$

When the moving sleeves close the slots, the inertia of the water in the gap between the resonator tube and the sleeve increases. The resulting dependence of the variable inductance $L^{*}$ on the displacement for a circular orifice can be approximately represented by Eq. (11), where $x$ is the displacement of the movable sleeve from the center position,

$$
L^{*}=\frac{\rho_{o}}{2} \sqrt{\frac{\pi}{A_{o}}}+\frac{\rho_{o}\left(0.5 l_{s}-x\right)\left(0.5 l_{s}+x\right)}{2 \pi r g l_{s}} .
$$

A short segment of a resonator tube (Fig. 5) with length $d X$ is simulated by an $L C$ resonant circuit, which can be described by a simple matrix equation

$$
\boldsymbol{V}_{n+1}=\boldsymbol{A} \boldsymbol{V}_{n},
$$

where

$$
\begin{aligned}
& \boldsymbol{V}_{n}=\left[\begin{array}{c}
U_{n} \\
I_{n}
\end{array}\right], \quad \boldsymbol{V}_{n+1}=\left[\begin{array}{c}
U_{n+1} \\
I_{n+1}
\end{array}\right], \\
& \mathbf{A}=\left[\begin{array}{cc}
1 & -i \omega L \\
-i \omega C & 1-\omega^{2} L C
\end{array}\right] .
\end{aligned}
$$

The admittance of the resonator tube can be calculated from the continued fraction.

$$
y=i \omega C_{b}+N^{2} /\left(1 /\left(i \omega C_{c}\right)+i \omega L_{c}+R_{t}+z\right),
$$

where

$$
\begin{aligned}
z= & i \omega L+1 /(i \omega C+1 /(i \omega L+\cdots 1 /(i \omega L+1 /(i \omega C \\
& +\frac{1}{i \omega L^{*}}+1 /(i \omega L+\cdots 1 /(i \omega C \\
& \left.\left.\left.\left.\left.\left.+\frac{1}{i \omega L_{m}+R}\right) \cdots\right)\right)\right) \cdots\right)\right) .
\end{aligned}
$$

The radiated volume velocity and pressure sound level $P_{\text {spl }}$ can be calculated by matrix equation

$$
\begin{aligned}
& U_{0}=U N, \\
& I_{1}=U_{0} /\left(1 /\left(i \omega C_{c}\right)+i \omega L_{c}+R_{t}+z\right), \\
& U_{1}=I_{1} z, \\
& \mathbf{V}_{1}=\left[\begin{array}{c}
U_{1} \\
I_{1}
\end{array}\right], \quad \mathbf{V}_{k}=\left[\begin{array}{c}
U_{k} \\
I_{k}
\end{array}\right], \\
& \mathbf{V}_{k}=\mathbf{A}^{k-k_{o}}\left[\begin{array}{cc}
\frac{-1}{i \omega L^{*}} & 1
\end{array}\right] \mathbf{A}^{k_{o}-1} \mathbf{V}_{1}, \\
& P_{\mathrm{spl}}=20 \log _{10}\left(\rho_{o} f\left|I_{k}\right|\right) .
\end{aligned}
$$

The simulation example of a tunable resonator tube was accomplished with the parameters listed in Table I.

The input projector voltage was $U=1500 \mathrm{~V}$. The dependence of the sound pressure level re $1 \mu$ pa at the distance of $1 \mathrm{~m}$ versus the position of the movable sleeve is shown in Fig. 6. The displacement of the sleeve was changed in $2 \mathrm{~mm}$ steps. The resonant frequency depends on a fluently changing sleeve position, and can be tuned to any frequency in the $200-300 \mathrm{~Hz}$ range. The $Q$ factor of the organ pipe was approximately 100 . The model gives a good qualitative assessment of the bandwidth and other resonator-tube projector properties. 
TABLE I. Piezoelectric-driver and resonator-tube parameters.

\begin{tabular}{|c|c|c|c|}
\hline \multirow{2}{*}{$\begin{array}{l}\text { Piezoelectric Tonpilz driver parameters } \\
\text { Length of one ceramic } \\
\text { cylinder } t_{c}(\mathrm{~m})\end{array}$} & \multicolumn{3}{|c|}{ Resonator tube parameters } \\
\hline & 0.0127 & Tube length $L(\mathrm{~m})$ & 1.397 \\
\hline Length of all the stacks $l_{c}(\mathrm{~m})$ & 0.0762 & Tube radius $R_{a}(\mathrm{~m})$ & 0.1683 \\
\hline $\begin{array}{l}\text { Entire area of the ceramic } \\
\text { stacks } A_{c}\left(\mathrm{~m}^{2}\right)\end{array}$ & 0.0081 & $\begin{array}{l}\text { Tube cross-sectional area } \\
\qquad A\left(\mathrm{~m}^{2}\right)\end{array}$ & 0.08899 \\
\hline Compliance $s_{33}^{E}\left(\mathrm{~m}^{2} / \mathrm{N}\right)$ & $18.5 \times 10^{-12}$ & Tube thickness $h(\mathrm{~m})$ & 0.0095 \\
\hline Electric permittivity $\varepsilon_{33}^{T}\left(\mathrm{~m}^{2} / \mathrm{N}\right)$ & $11510.2 \times 10^{-12}$ & Orifice distance $L_{0}(\mathrm{~m})$ & 0.4572 \\
\hline $\begin{array}{l}\text { Piezoelectric strain } \\
\text { coefficient } d_{33}(\mathrm{C} / \mathrm{N})\end{array}$ & $253 \times 10^{-12}$ & Orifice area $A_{0}\left(\mathrm{~m}^{2}\right)$ & 0.0768 \\
\hline Ceramic density $\rho_{c}\left(\mathrm{~kg} / \mathrm{m}^{3}\right)$ & 7500 & Gap under sleeve $g(\mathrm{~m})$ & 0.002 \\
\hline Pipe length $l_{p}(\mathrm{~m})$ & 0.06985 & Coaxial sleeve length $l_{s}(\mathrm{~m})$ & 0.02 \\
\hline Material (aluminum) density $\rho_{p}\left(\mathrm{~kg} / \mathrm{m}^{3}\right)$ & 2700 & Water density $\rho_{0}\left(\mathrm{~kg} / \mathrm{m}^{3}\right)$ & 1005 \\
\hline Piston area $A_{p}\left(\mathrm{~m}^{2}\right)$ & 0.061311 & Sound velocity $c_{0}(\mathrm{~m} / \mathrm{s})$ & 1490 \\
\hline $\begin{array}{l}\text { Number of piezoelectric } \\
\text { ceramic cylinders } n\end{array}$ & 6 & $\begin{array}{l}\text { Compressibility of water } \\
\qquad \beta\left(\mathrm{m}^{2} / \mathrm{N}\right)\end{array}$ & $4.9416 \mathrm{e}-01$ \\
\hline Number of ceramic stacks $m$ & 4 & Young modulus $E\left(\mathrm{~N} / \mathrm{m}^{2}\right)$ & $6.895 e+09$ \\
\hline
\end{tabular}

\section{TUNING PROCESS SIMULATION}

The electrical circuit model can be used to simulate the resonant-tube tuning process. In a time domain analysis, the model (Fig. 5), can be rewritten as a differential equation system for capacitor voltages and inductance currents. Note that voltages $\widetilde{U}_{1}(t), U_{1}(t)$ are measured in different places on the circuit, and resistor $R_{t}$ is not taken into account,

$$
\begin{aligned}
& \frac{d \widetilde{U}_{1}(t)}{d t}=\frac{d U_{0}(t)}{d t}-\frac{1}{C_{c}} I_{1}(t), \\
& \frac{d I_{1}(t)}{d t}=\frac{1}{L_{c}+L}\left(\widetilde{U}_{1}-U_{0}\right), \\
& \frac{d U_{2}(t)}{d t}=\frac{1}{C}\left(I_{1}(t)-I_{2}(t)\right), \\
& \frac{d I_{2}(t)}{d t}=\frac{1}{L}\left(U_{2}-U_{3}\right), \\
& \frac{d U_{3}(t)}{d t}=\frac{1}{C}\left(I_{2}(t)-I_{3}(t)\right), \\
& \vdots, \\
& \frac{d I_{k 0}(t)}{d t}=\frac{1}{L}\left(U_{k 0}-U_{k 0+1}\right)+\frac{1}{L^{*}} U_{k 0}, \\
& \frac{d I_{k 0}^{*}(t)}{d t}=\frac{1}{L}\left(U_{k 0}-U_{k 0+1}\right), \\
& \frac{d U_{k 0+1}(t)}{d t}=\frac{1}{C}\left(I_{k 0}^{*}(t)-I_{k 0+1}(t)\right), \\
& \frac{d I_{k}(t)}{d t}=\frac{1}{L_{r}} U_{k}(t)-\frac{R}{L_{r}} I_{k}(t)
\end{aligned}
$$

The radiated volume velocity and sound pressure level are represented by the output current $I_{k}(t)$.

The implicit Crank-Nicholson method was used for numerical simulation of the ordinary differential equation system. Figure 7 presents the solutions.

The instantaneous frequency hop is presented in Figs. $7(a)$ and 7(b). The exciting signal is shown in gray. This signal is shifted $90^{\circ}$ from the output because of the capacitor $C_{c}$. The signal maintains the same phase relative to the output signal during the entire process. The frequency shift in the reference signal is $100 \mathrm{~Hz}$. The orifice area was instantaneously opened to provide a corresponding change in the organ-pipe resonant frequency. Engineering such a fast acoustical vent presents a special technological problem that is not considered in this paper. This type of acoustical modulation can be accomplished, for example, by blocking movable piston vibrations using magneto-rheological liquid or other technologies. ${ }^{11-18}$ The purpose of this research is to demonstrate the possibility of such an approach. The simulation shows that simultaneously changing the excitation sig-
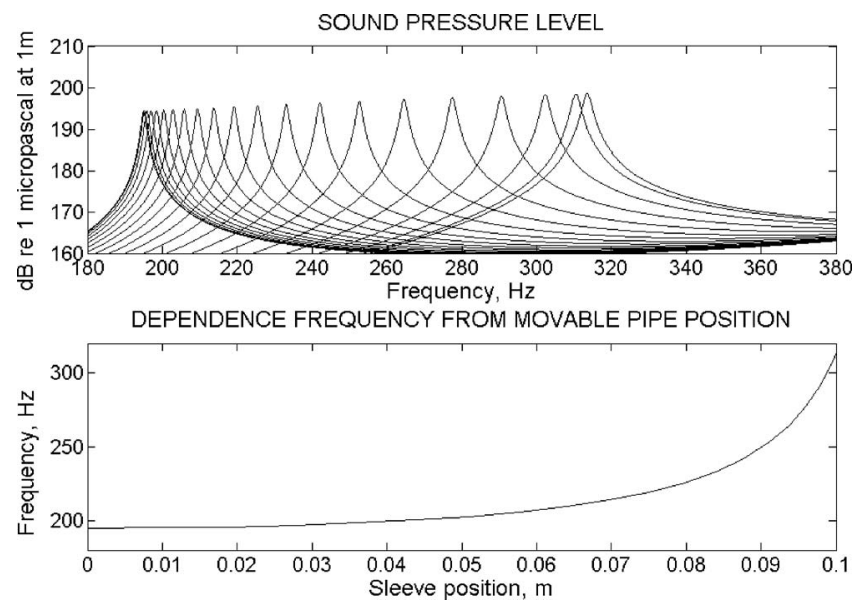

FIG. 6. The sound pressure level of a tunable resonator tube for different positions of a movable sleeve; the difference between any two displacements is $2 \mathrm{~mm}$. 

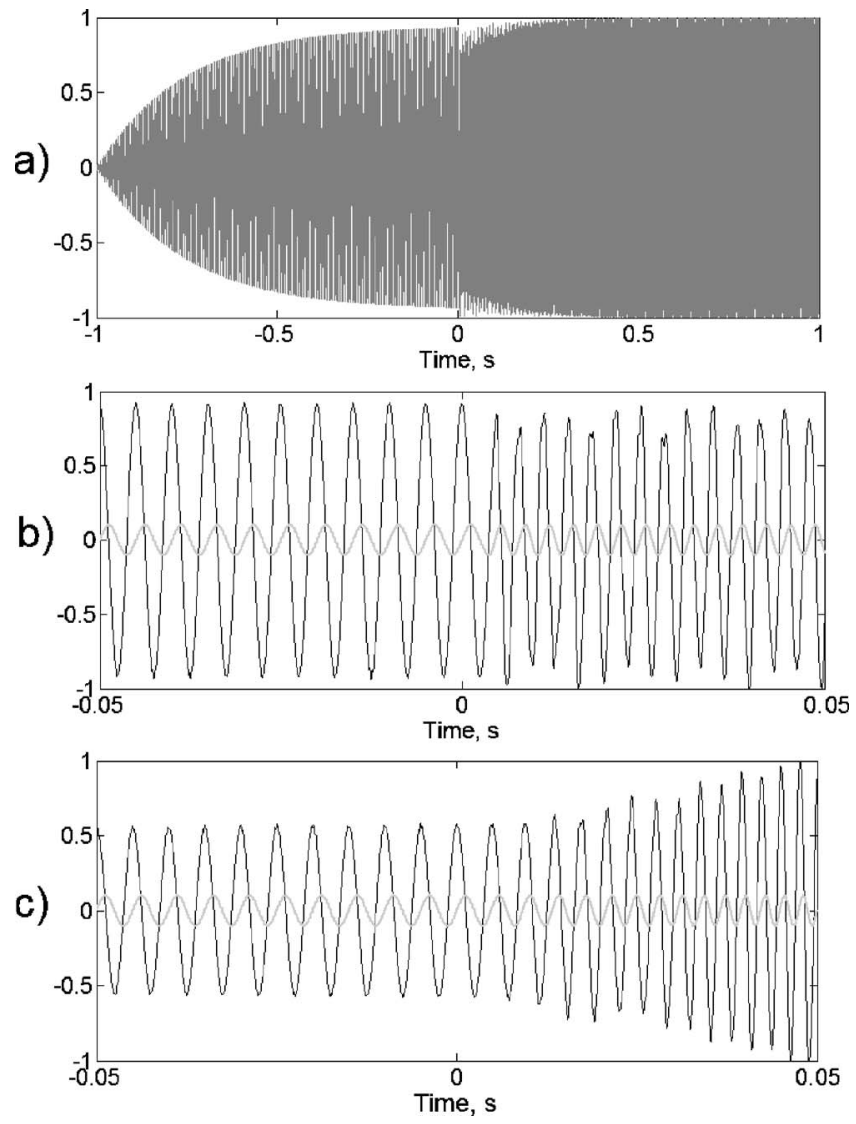

FIG. 7. (a) The transient response for a frequency hop from 200 to $300 \mathrm{~Hz}$ at time moment $t=0$. (b) The transient response of a tunable organ pipe for a frequency hop from 200 to $300 \mathrm{~Hz}$ at time moment $t=0$, large time scale. (c) The tunable organ pipe frequency sweeping from 200 to $300 \mathrm{~Hz}$ for $50 \mathrm{~ms}$.

nal and the resonance frequency of a tunable organ pipe leads to a quick change in the radiated wave form. The difference with a Helmholtz resonator is an irrelevant transient process in high-frequency resonance harmonics. The highfrequency response process is approximately $100 \mathrm{~ms}$, with amplitude fluctuations of about $10 \%$. However, the high- $Q$ organ pipe retains the ability for quick frequency hopping.

The same conclusion applies to fast frequency sweeping. A sweep from 200 to $300 \mathrm{~Hz}$ was simulated with the same equation system (15) and presented in Fig. 7(c). There is a small transient response to the sweeping of the sound source frequency from 200 to $300 \mathrm{~Hz}$ for $50 \mathrm{~ms}$. These transient fluctuations can be easily corrected using phase locked loop.

\section{EXPERIMENTAL TESTING OF TUNABLE ORGAN PIPES}

Three identical, tunable, organ-pipe projectors with frequency swept signals were built for ocean-acoustic tomography and long-range sound propagation experiments in spring 2004. During manufacture, the wall thickness was changed from $0.0095 \mathrm{~m}$, as was used during simulation, to $0.0125 \mathrm{~m}$. As a result, the resonance frequency increased to $25 \mathrm{~Hz}$ and sources were sweeping from 225 to $325 \mathrm{~Hz}$. The prototype source manufactured with a $0.0095 \mathrm{~m}$ wall ${ }^{20}$ swept in the expected $200-300-\mathrm{Hz}$ frequency band. Unfortunately, the first prototype used an actuator with a very low rate, which

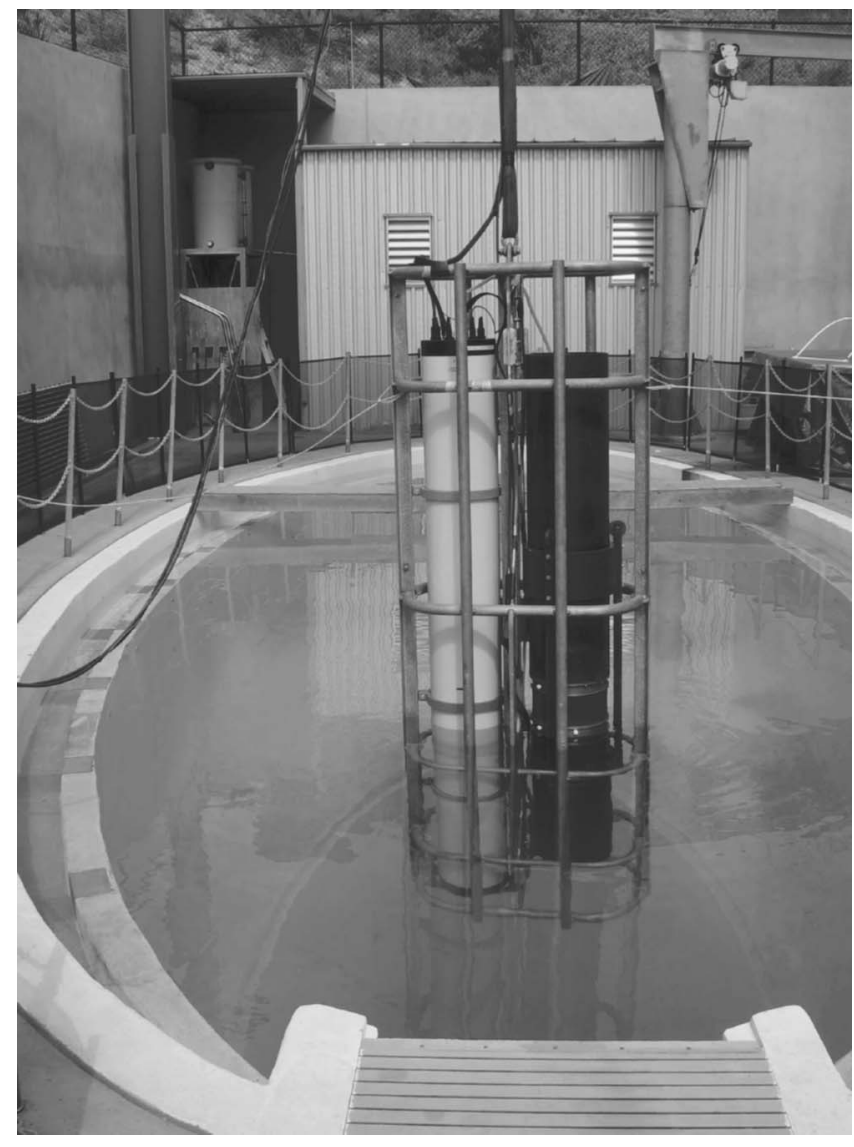

FIG. 8. Low frequency deepwater sound source in the SCRIPPS testing pool.

did not allow testing of high-rate frequency sweeping. The system used a tunable organ-pipe resonator with an electromechanical actuator and interior hydrophone, which were combined into a PLL controlled circuit. The linear mechanical actuator moved the coaxial sleeve to maintain resonance with the instantaneous frequency of the signal transmitted. The system was built for long-term ocean monitoring in deep water $(\sim 5000 \mathrm{~m})$, and it was equipped with a battery set, a low-consumption controller, a hybrid rubidium clock, and an acoustic navigation system. Figure 8 is a recent photo of the sound-source system in the Scripps Institution of Oceanography test pool. Before deployment in 2004, the system was tested in the Seneca Lake Sonar Test Facility. The results of that test are presented in the following.

Although three sound sources were built with approximately equal parameters, only one of them will be considered in this paper. The complete sound-source system, connected to a standard measurement system, was submerged in a vertical position to $95.4 \mathrm{~m}$ below the surface platform. The hydrophone was located $11.41 \mathrm{~m}$ from the source and at the same depth.

The main parameter of the long-term system is its efficiency. The test shows that the tunable organ pipe has the expected high efficiency of an ordinary organ pipe and approximately the same directivity with $3 \mathrm{~dB}$ gain in a vertical plane. In a horizontal plane, the source, with attached rigid electronics housing, is omnidirectional. Figure 9 shows the efficiency of the sound source. Note that this efficiency was 


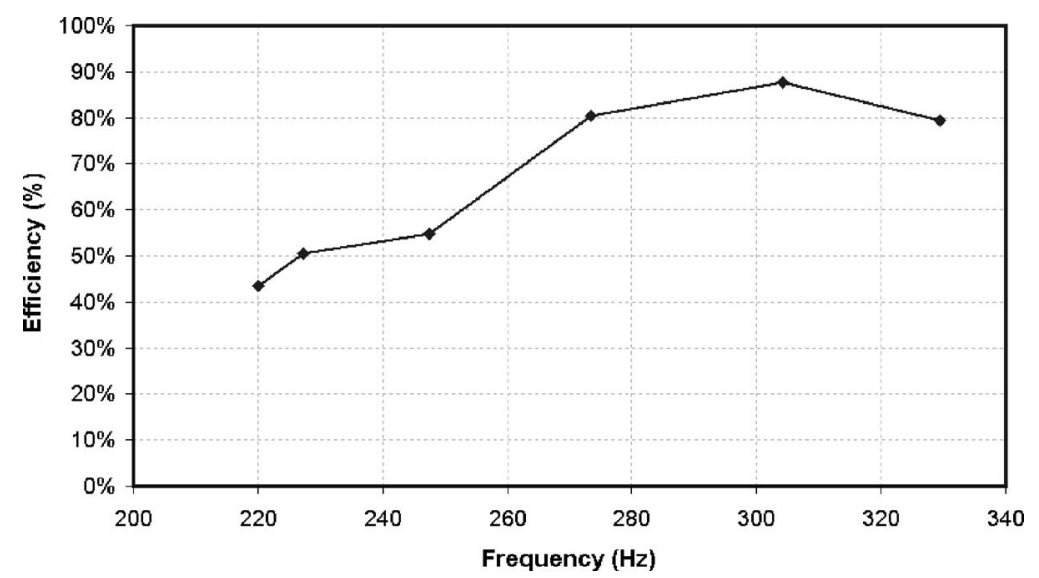

FIG. 9. The efficiency of sound source vs frequency in an isotropic approximation.

measured by an automatic test system, which plots the efficiency for omnidirectional or isotropic projectors. The actual organ pipe was closer to the dipole than to the omnidirectional source and, hence, its real efficiency was smaller. Nevertheless, even after correction for directivity, the efficiency of the source is very high.

The sound-source system incorporated a powerful Tecnadyne Inc. linear actuator capable of a $5 \mathrm{~cm} / \mathrm{s}$ maximum stroke rate. To sweep the frequency over $100 \mathrm{~Hz}$, the actuator needed to shift the sleeve $10 \mathrm{~cm}$. To save power the actuator was used at one-third of its rated voltage; nevertheless, it could move $8 \mathrm{~cm}$ in approximately $3 \mathrm{~s}$, allowing us to test for high-rate sweeping and to check the above-described theory.

We conducted sound-source testing for three different durations of $100 \mathrm{~Hz}$ frequency sweeping: 135, 10, and $5 \mathrm{~s}$. All tests were done with the PLL system active. Transmission of each broadband 225 to $325 \mathrm{~Hz}$ swept frequency signal begins with a section of CW signal with a $225 \mathrm{~Hz}$ carrier frequency. This signal is used to adjust the resonant frequency of the system to the start position before frequency sweeping begins. The PLL feedback continues to function over the period of the swept signal transmission, keeping the resonant frequency in compliance with the instantaneous signal frequency. Figure 10 shows the spectrogram of the three signals with the different frequency-sweeping rates. The sig-

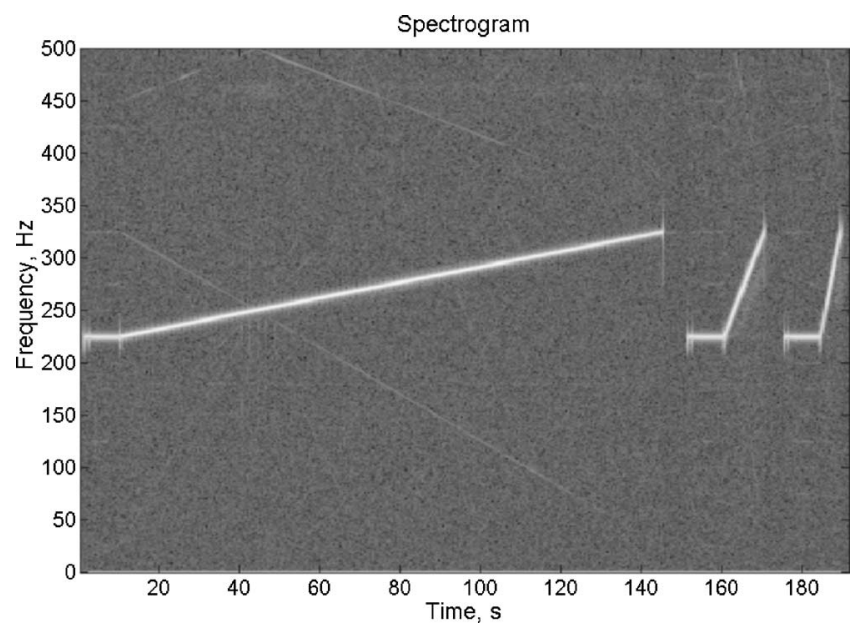

FIG. 10. Spectrogram for 135,10 , and $5 \mathrm{~s}$ sweep over $100 \mathrm{~Hz}$ bandwidth. nals were transmitted in a series with a small interval between them. The flat part of the spectrogram plots the start position's adjustment period.

The correlation functions between the reference signal and the actual transmitted signal are shown in Fig. 11. A solid line plots theoretical dependence in Fig. 11, which demonstrates very good agreement between theoretical and experimental correlations. The internal digital controller analyzed and recorded PLL error, which was only a few degrees. The error increased for the initial part of $5 \mathrm{~s}$ frequency sweeping, which explains the difference in theoretical and experimental correlations in Fig. 11. This initial PLL error increased when the test was run for a $3 \mathrm{~s}$ sweep. The PLL worked for the $3 \mathrm{~s}, 100 \mathrm{~Hz}$ frequency swept signal but exhibited a large phase error at the beginning that resulted in a decrease in amplitude. The effect can be explained by the movable sleeve's inertia. A more powerful motor was needed to move the heavy sleeve at the necessary speed. Nevertheless, after 1-1.5 s of acceleration, the sleeve reached the necessary position and speed, and it worked well during the last part of the signal. Based on our analysis and experimental research, we conclude that, with a light sleeve made of composite carbon-fiber materials, the system can provide frequency sweeping in a $100 \mathrm{~Hz}$ band for a fraction of a second. After testing in Seneca Lake, two deep-water sound sources were successfully used in the SPICE04 and LOAPE (2004) experiments.

\section{CONCLUSION}

Computer simulation and field testing of a lowfrequency sound source with a variable resonant frequency shows that this design is highly efficient and exhibits significant ability for fast frequency change. A resonant tube sound source with a computer-controlled resonant frequency can be used for radiating broadband swept frequency signals at the rate of $100 \mathrm{~Hz}$ for a few seconds and with a lighter sleeve for a fraction of a second. Computer control enables holding the resonant frequency in compliance with the instantaneous signal frequency with a very small error. The correlation function of the linear frequency modulated signal is very close to the theoretical one. The frequency bandwidth of such a projector can reach a value of 0.7 to 0.8 of the central frequency. This sound-projector system is easily deployed and can op- 

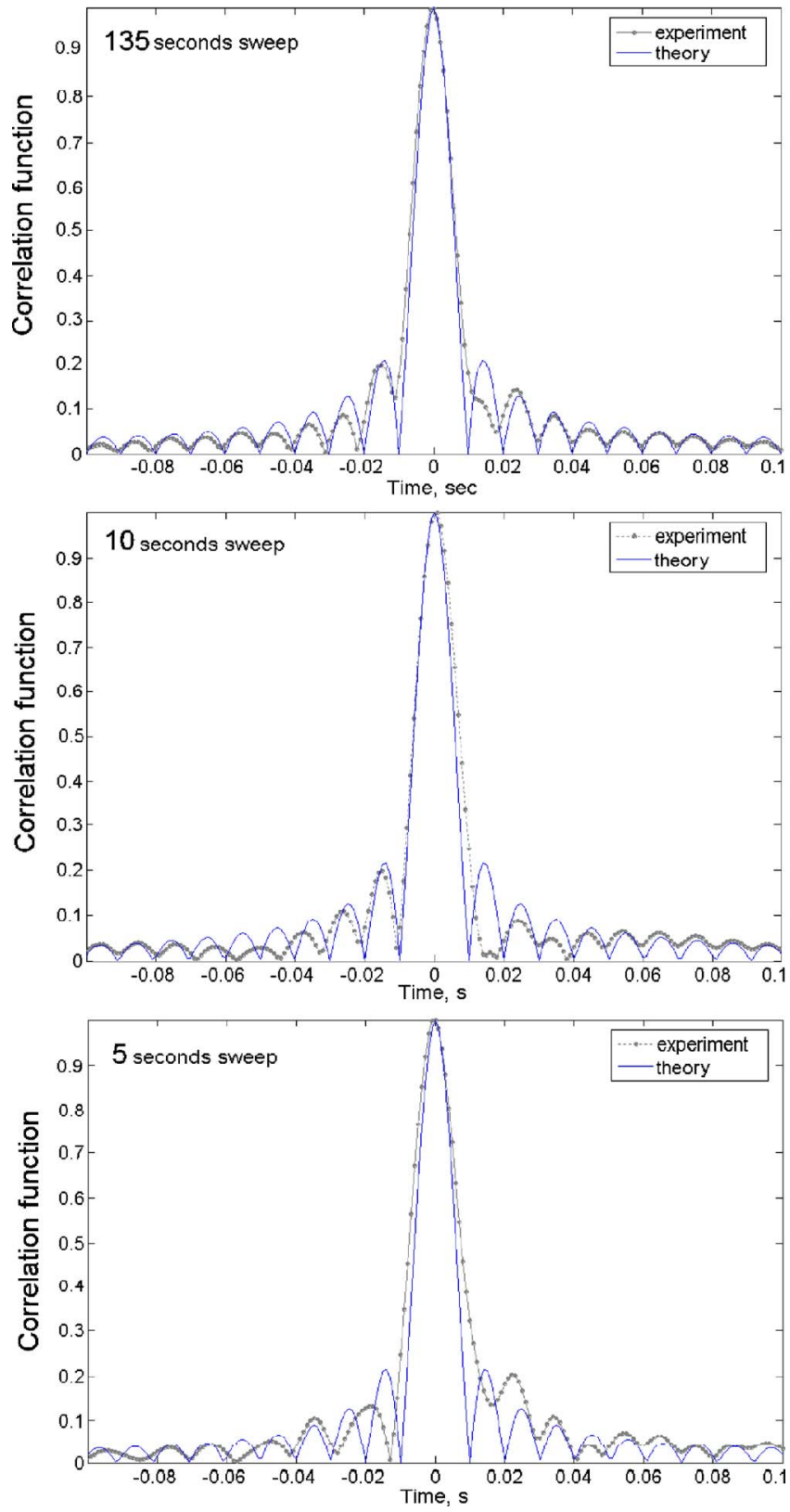

FIG. 11. (Color online) Correlation function for 135,10 , and $5 \mathrm{~s}$, 225-325 Hz frequency sweep. Seneca Lake Sonar Test Facility, 3 May 2004.

erate on alkaline batteries for a long-term period. It is recommended for deep-water research, such as ocean-acoustic tomography and deep-penetration seismic profiling.

\section{ACKNOWLEDGMENTS}

The authors gratefully acknowledge the support and help of Dr. P. Worcester, Dr. W. Munk from Scripps Institution of Oceanography, and T. Ensign, the president of Engineering Acoustics, Inc., Orlando, FL. The work was supported by ONR.
${ }^{1}$ W. H. Munk and C. Wunsch, "Ocean acoustic tomography: A scheme for large-scale monitoring," Deep-Sea Res., Part A 26, 123-161 (1979).

${ }^{2}$ P. F. Worcester, R. C. Spindel, and B. W. Howe, "Reciprocal acoustic transmissions: Instrumentation for mesoscale monitoring of ocean currents," IEEE J. Ocean. Eng. 10, 123-137 (1985).

${ }^{3}$ G. R. Potty, J. H. Miller, J. F. Lynch, and K. B. Smith, "Tomographic inversion for sediment parameters in shallow water," J. Acoust. Soc. Am. 108, 973-986 (2000).

${ }^{4}$ T. H. Ensign and D. C. Webb, "Electronic performance modeling of the gas-filled bubble projector," Proceedings of the Third International Workshop on Transducers for Sonic and Ultrasonics, 6-8 May, Orlando, FL, pp. $268-275,1992$

${ }^{5}$ R. S. Woollett, "Basic problems caused by depth and size constraints in low-frequency underwater transducers," J. Acoust. Soc. Am. 68, 10311037 (1980).

${ }^{6} \mathrm{O}$. B. Wilson, Introduction to the Theory and Design of Sonar Transducers (Peninsula, Los Altos, 1988).

${ }^{7}$ G. W. McMahon, "Performance of open ferroelectric ceramic rings in underwater transducers," J. Acoust. Soc. Am. 36, 528-533 (1964).

${ }^{8}$ J. B. Lee, "Low-frequency resonant-tube projector for underwater sound," in Proceedings IEEE Ocean'74, Nova Scotia, Halifax, 21-23 August, Vol. 2, pp. 10-15, 1974.

${ }^{9}$ T. J. Rossby, J. Ellis, and D. C. Webb, "An efficient sound source for wide area RAFOS navigation,” J. Atmos. Ocean. Technol. 10, 397-403 (1993).

${ }^{10}$ G. D. Larson, P. H. Rogers, and W. Munk, "State switched transducers: A new approach to high-power, low-frequency, underwater projectors," J. Acoust. Soc. Am. 103, 1428-1441 (1998).

${ }^{11}$ H. A. B. Alwi, J. R. Carey, and B. V. Smith, "Chirp response of an active-controlled thickness-drive tunable transducer," J. Acoust. Soc. Am. 107, 1363-1373 (2000).

${ }^{12}$ G. A. Steel, B. V. Smith, and B. K. Gazey, "Tunable sonar transducer," Electron. Lett. 22, 758-759 (1986).

${ }^{13}$ G. A. Steel, B. V. Smith, and B. K. Gazey, "Active electronic-control of the response of a sonar transducer," Proc. Inst. Acoust. 9, 79-87 (1987).

${ }^{14}$ S. K. Jain and B. V. Smith, "Tunable sandwich transducer," Electron. Lett. 24, 311-312 (1988).

${ }^{15} \mathrm{~W}$. Chenghao and Z. Zheying, "Principle of piezoelectric-tunable transducer," Chin. J. Acoust. 2, 16-24 (1983).

${ }^{16}$ B. A. Kasatkin and N. Y. Pavin, "Piezoelectric transducer with controlled response characteristics," Sov. Phys. Acoust. 29, 418-419 (1983).

${ }^{17}$ H. A. B. Alwi, B. V. Smith, and J. R. Carey, "Tunable transducers," Proc. Inst. Acoust. 17, 173-182 (1995).

${ }^{18}$ H. A. B. Alwi, B. V. Smith, and J. R. Carey, "Factors which determine the tunable frequency range of tunable transducers," J. Acoust. Soc. Am. 100, 840-847 (1996).

${ }^{19}$ D. C. Webb, A. K. Morozov, and T. H. Ensign, "A new approach to low frequency wide-band projector design," Proceedings of Oceans, 2002, pp. 2342-2349.

${ }^{20}$ A. K Morozov and D. C. Webb, "A sound projector for acoustic tomography and global ocean monitoring," IEEE J. Ocean. Eng. 28, 174-185 (2003).

${ }^{21}$ A. K. Morozov and D. C. Webb, "Underwater sound source with tunable resonator for ocean acoustic tomography," J. Acoust. Soc. Am. 116, 2635 (2004).

${ }^{22}$ L. D. Ambs and J. J. Sallas, "Marine seismic source," J. Acoust. Soc. Am. 110, 651 (2001).

${ }^{23}$ F. Liu, S. B. Horowitz, T. Nishida, L. N. Cattafesta, and M. Sheplak, "A tunable electromechanical Helmholtz resonator," Ninth AIAA/CEAS Aeroacoustics Conference and Exhibit 2003.

${ }^{24}$ M. Sheplak, L. Cattafesta, T. Nishida, and S. B. Horowitz, U. S. Patent No. 6,782,109, 2004.

${ }^{25}$ C. B. Birdsong and C. J. Radcliffe, "A compensated acoustic actuator for systems with strong dynamic pressure coupling," J. Vibr. Acoust. 121, 89-94 (1999).

${ }^{26}$ K. Nagaya, Y. Hano, and A. Suda, "Silencer consisting of two-stage Helmholtz resonator with auto-tuning control," J. Acoust. Soc. Am. 110, 289295 (2001).

${ }^{27}$ B. L. Fanning and G. W. McMahon, U.S. Patent No. 4,855,964, 8 July 1988. 